\title{
Maintaining item banks with the Rasch model: An example from wave optics
}

\author{
Džana Salibašić Glamočić $\odot,{ }^{1,}{ }^{*}$ Vanes Mešić $\odot,{ }^{1}$ Knut Neumann, ${ }^{2}$ Ana Sušac $\odot,{ }^{3}$ \\ William J. Boone $\odot,{ }^{4}$ Ivica Aviani, ${ }^{5}$ Elvedin Hasović, ${ }^{1}$ Nataša Erceg $\odot,{ }^{6}$ \\ Robert Repnik $\oplus^{7}$ and Vladimir Grubelnik $\oplus^{8}$ \\ ${ }^{1}$ Faculty of Science, University of Sarajevo, \\ Zmaja od Bosne 33-35, 71000 Sarajevo, Bosnia and Herzegovina \\ ${ }^{2}$ Leibniz Institute for Science and Mathematics Education (IPN) at the University of Kiel, \\ 24118 Kiel, Germany \\ ${ }^{3}$ Faculty of Electrical Engineering and Computing, University of Zagreb, \\ Unska ul. 3, 10000 Zagreb, Croatia \\ ${ }^{4}$ Department of Educational Psychology, Miami University, 201 McGuffey Hall, Oxford, Ohio 45056, USA \\ ${ }^{5}$ Department of Physics, Faculty of Science, University of Split, \\ Ulica R. Boškovića 33, 21000 Split, Croatia \\ ${ }^{6}$ University of Rijeka, Department of Physics, R. Matejčić 2, 51000 Rijeka, Croatia \\ ${ }^{7}$ Faculty of Natural Science and Mathematics, University of Maribor, \\ Koroška cesta 160, 2000 Maribor, Slovenia \\ ${ }^{8}$ Faculty of Electrical Engineering and Computer Sciences, Koroška cesta 46, 2000 Maribor, Slovenia
}

(Received 10 June 2020; accepted 8 January 2021; published 5 February 2021)

\begin{abstract}
Item banks are generally considered the basis of a new generation of educational measurement. In combination with specialized software, they can facilitate the computerized assembling of multiple preequated test forms. However, for advantages of item banks to become fully realized it is important that the item banks store a relatively large number of valid test items. In this paper, we demonstrate how the Rasch model is used for integrating new items into an existing wave optics item bank. First, we identified and applied a set of criteria for selecting 18 linking items from our initial item bank. In order to integrate 12 newly developed items, we combined the 18 linking items with the 12 newly developed ones into one test and administered this test to 106 postinstruction physics students from 4 universities in Slovenia, Croatia, and Bosnia and Herzegovina. It was determined that the 12 new items measure the same construct as items from the initial item bank. In addition, all items exhibited good item fit, item reliability was excellent and person reliability was fair. The ratio of standard deviations of linking item difficulties for the new test and existing item bank amounted to 0.89 and correlation of these difficulties amounted to 0.93 which indicated good linking precision. We could conclude that good linking precision can be obtained if linking items are chosen based on the following set of criteria: number of items, item fit, range and spacing of item difficulties, content representativeness, position in test form and interuniversity DIF contrasts.
\end{abstract}

DOI: 10.1103/PhysRevPhysEducRes.17.010105

\section{INTRODUCTION}

In student-centered approaches to physics teaching it is very important to continually collect information about our students' conceptual understanding. Teachers and researchers often collect this information by having the students answer conceptual questions. Thereby, the quality of collected information largely depends on the quality of

*sdzana@hotmail.com

Published by the American Physical Society under the terms of the Creative Commons Attribution 4.0 International license. Further distribution of this work must maintain attribution to the author(s) and the published article's title, journal citation, and DOI. the questions. For collecting reliable and valid information about understanding in students of different ability levels it is important to have at our disposal a large number of questions that function together to reflect various levels of that understanding. This is exactly what we have in item banks.

An item bank may be defined as a "composition of coordinated questions that develop, define and quantify a common theme and thus provide an operational definition of a variable" [1]. As such, "item banks" should be distinguished from "item pools" which also are collections of items grouped by a common theme but typically these items have not undergone calibration [2].

For each item, a bank includes a large amount of information, such as information about its psychometric 
features, its assignment to certain assessment goals, and comments on how the item functioned in the past [2-4].

Generally, item banks may be used for generating different sets of items, i.e., test forms, that are in line with some assessment specifications [5,6]. This feature is utilized in computer adaptive testing (CAT) which allows for cost effective and reliable assessment of each individual student's learning [7,8]. The items in adaptive tests are chosen from the item bank to match the estimated student ability. If the student answers the item correctly, the next item is a little more challenging. After an incorrect answer, an easier item is presented. By choosing items of appropriate difficulty as the student progresses through the test, student's ability can be determined rather quickly and to the required accuracy.

Item banks also can facilitate conducting high-quality experimental research. In physics education research (PER), typically, the same research-based instrument is administered to all students from a sample as pretest and post-test to provide a standardized measure of students' learning. However, one instrument cannot measure with the same precision the students' ability in the pretest and posttest contexts. Therefore, recently it has been suggested to use two different instruments for pretest and post-test. This may ensure better test targeting for both pretest and posttest samples and reduce the impact of students remembering items which were presented for a pretest [9]. Again, item banks would make the construction of comparable pretest and post-test instruments much easier, because in item banks all items are on the same scale.

It is useful to distinguish the process of developing and of maintaining item banks. When it comes to developing item banks, the process is very similar to developing tests. Typically, this process starts with defining the construct of interest, after which the construct is delineated into items and item functioning is tested through small-scale and large-scale studies [10]. After developing an item bank, we have to continually reflect upon whether our set of items is still a valid and reliable measure of the given construct. The process of maintaining item banks thus involves depositing new items (i.e., adding new items into the item bank), removing obsolete items, and protecting the security of the item bank $[3,11]$.

A powerful technique for developing and maintaining item banks is the Rasch analysis [12]. Rasch analysis is based on the idea that there exists a measure common to both items and examinees [13]. This measure is expressed on an interval scale that describes a continuum of increasing competency on the corresponding construct and is sometimes also referred to as a linear scale [14]. Consequently, use of Rasch model based item banks may facilitate construct-based interpretation of students' scores and provide interval measures of achievement that lend themselves to being used in typical parametric tests such as analysis of variance (ANOVA) [14].
Furthermore, the Rasch model is very practical for linking scales and identifying misfitting items, which proves to be important in item depositing and removing of obsolete items. In addition, combining Rasch analysis with test assembly software, such as FastTest, makes it easier to systematically assemble test forms in line with predefined psychometric features [15]. One comparative advantage of Rasch analysis over other approaches is that it works well even with small sample sizes; for item banking, samples of 100 persons are considered sufficient [16].

Although the use of item banks is associated with many potential benefits, in PER the potential of item banks is still not sufficiently utilized. In our recent study [10] we described the development of an initial wave optics item bank, and in this study our aim is to demonstrate another important activity in item banking, which is depositing new items in the initial item bank. Together with the paper by Mešić et al. [10], this paper will therefore provide a systematic overview of the processes of developing and maintaining item banks within the Rasch framework. Taking into account that existing PER literature lacks papers that thoroughly discuss the procedures of developing and maintaining item banks, we hope that our paper may contribute to methodological advancement of PER. By demonstrating the process of developing and maintaining item banks in the authentic context of wave optics, we hope to motivate other physics education researchers to utilize the opportunities that are associated with the use of item banks, such as computer adaptive testing and generating comparable pretest and post-test instruments in the context of experimental research. Eventually, this could contribute to higher-quality measurements which may lead to higherquality research. Besides its contribution to methodology of physics education research, our paper may also represent a valuable contribution to classroom practice, because it provides some original, extensively validated conceptual questions that may be used for uncovering students' misconceptions and provoking classroom discussions about wave optics phenomena.

\section{THEORETICAL BACKGROUND}

The quality of assessments is commonly determined through objectivity, reliability, and validity of measurement. Objectivity refers to the extent to which results of measurement depend on our choice of the person who administers the assessment instrument and scores students' answers $[17,18]$. Using item banks in combination with test assembly software may help us to improve objectivity, by allowing computerized assembling and administering of test forms, as well as automatized scoring and reporting of students' scores $[2,15]$. Reliability is commonly measured by the (internal) consistency of measurement and it can be maximized around a certain cutoff by utilizing the Rasch approach with item banks [8]. Finally, item banks may also help us to accomplish valid measurement by facilitating the assembly of test forms that appropriately reflect the 
measured construct. However, in order to make the fullest use of the potential of item banks, it is necessary that an item bank contains a large number of high-quality items. Managing the quality and number of items in our item banks proceeds through the processes of developing, using, and maintaining item banks.

\section{A. Developing and using item banks}

As noted earlier, item banks are compositions of coordinated items that define a common theme [1]. They typically contain the following information [2-4]: (a) item ID, (b) item text, (c) correct answer, (d) classification with respect to content area, learning goal or item type, (d) target population, (e) psychometric features (e.g., difficulty), (f) author, source and date of creation, and (g) comments on how the item functioned in the past.

Item bank development may proceed through a priori or a posteriori approaches [10,19-21]. In a priori approaches, one starts from a definition of the construct and operationalizes it through learning objectives which help to delineate the construct into items. In the next step, small-scale item try outs are conducted (e.g., think alouds and expert surveys), which is followed by large-scale field testing and calibration procedures. On the other hand, in a posteriori approaches, researchers first identify a number of different instruments that are supposed to measure a single construct, and then they conduct linking studies for purposes of bringing items from different instruments onto a single scale. Often this is followed by validation studies supporting the assertion that it is appropriate to combine items from different instruments for measuring a given construct.

One of the fields in which item banking is frequently utilized is health research. In one of the many item banking studies that were funded by the National Institute of Health, Pesudovs [19] used the Rasch model for combining items from 19 visual related activity limitation (VRAL) instruments into a single VRAL scale. Similarly, Smith et al. [20] reported about combining eight psychological distress instruments for developing a bank of 63 items that measure psychological distress in cancer patients. Evidently, both of these studies followed an a posteriori approach to item bank development. There are also examples of a priori approaches to item banking in which researchers developed assessment goals and/or test items on their own. For example, Sadler et al. [22] describe the development of a life sciences item bank based on K-8 science standards. The researchers constructed $476 \mathrm{MC}$ items and used them for assessment of teachers' and students' knowledge. Similarly, Cupani et al. [23] used the Rasch model to develop an item bank for measuring knowledge of biology at the University of Cordoba. Altogether, 180 items have been banked for computerized adaptive testing applications.

Unlike in health research and life sciences, to the best of our knowledge, there are not many research papers reporting upon the development and use of item banks in physics education. Mostly, there are studies in which physics item banks were developed by Rasch calibration of existing item pools. Concretely, Mestre, Morphew, and Gladding [24] carefully selected problems from previously administered exams to develop an item bank consisting of 222 physics items. This bank was then used for purposes of cognitivediagnostic computer adaptive testing. Similarly, Njiru and Romanoski [21] report about Rasch calibrating physics items from the Tertiary Entrance Exam in Western Australia to create a physics item bank. Misfitting items were dropped or revised and eventually an item bank consisting of 174 items has been created, which can be used by teachers for practice in their classrooms or for developing assessments of student achievement. In other studies, an a priori, construct driven approach has been followed. For example, Ene and Ackerson [25] applied the Rasch model to calibrate a 30 item unidimensional scale for measuring students' conceptual knowledge about semiconductors and they showed that the developed scale can be used as an item bank from which short testlets may be extracted. Another a priori study has been conducted by Mešić et al. [10]. Based on a theoretical model of understanding of wave optics, Mešić et al. [10] developed an item pool consisting of 65 wave optics items. The quality of these items was checked through content-expert (content validity) surveys, think-alouds, and focus group interviews (cognitive validity), as well as through written item try outs. Thereafter, Mešić et al. [10] field tested 35 out of these 65 items on a sample of 188 physics and engineering students from five universities from Slovenia, Croatia, and Bosnia and Herzegovina. Taking into account that many items exhibited large DIF contrast for comparisons of physics and engineering students, Mešić et al. [10] eventually suggested the items be used with physics students only. For the physics students subsample, 32 items were shown to fit the model and reflect a single construct. These 32 items constituted the initial bank of wave optics items.

Compared to item banking, in the physics education research community, more studies were conducted on the related topic of test linking and developing short forms of measurement instruments. For example, Han et al. [26] developed two half-length versions of the FCI and validated the assessment equivalence between the full-length version and two half-length versions of the instrument. Then, Xiao et al. [27] extended Han's algorithm for developing short and parallel CI's with an emphasis on incorporating standard practices in test equating. They used the two-parameter logistic item response model to develop and validate short forms of BEMA and linked the scores between BEMA and CSEM, thus allowing comparability of BEMA and CSEM scores.

\section{B. Maintaining item banks in physics education}

An item bank must be kept as a living bank with continual editing, updating, and refining [28]. For that purpose, we have to continually collect and analyze 
information about item functioning in different testing occasions and with different (sub)populations of respondents. In this quality management process, existing items may be rewritten or dropped, and some new items may be added.

For example, it is important to periodically check whether the items from our item bank continue to validly measure the given construct or at some point they begin to measure another construct $[29,30]$. In fact, the extent to which an item validly measures a construct depends not only on the item but also the respondents. This touches the issue of sample independence. Although it is common thinking that items aligning with the Rasch model are sample independent, this is limited to any sample from a population the item has been designed for and the Rasch model applies to [17,31]. An item may stop validly measuring a construct, for example, if the respective content is removed from the curriculum, potentially removing respective learning opportunities. In addition, relative item difficulties may change over time and an update of item difficulties may be necessary [29]. Similarly, some items may lose their relevance or may be in contradiction with recent scientific conventions (e.g., naming Pluto as a planet). In other cases, we could come to the conclusion that a specific item has been used in too many testing occasions, and it should be "retired" before its overexposure leads to nonvalid conclusions about students' learning [30]. Generally, an important aspect of item bank quality management is to check whether the composition of the item bank is in line with the assessment blueprint and whether the assessment blueprint is in line with the curriculum. Thereby, we often decide that new items have to be added for purposes of replacing dropped items or achieving a better alignment with the curriculum.

We can effectively manage the quality of item banks by using the Rasch model $[12,14,32]$. This model may help us to evaluate whether items from our bank continue to work well together to measure a single construct, as well as to inspect differential functioning of items or groups of items in different populations of respondents. In addition, Rasch analysis allows us to inspect the empirical hierarchy of item difficulties which may be compared with our theoretical model of the measured construct. Whether we use Rasch analysis or not, an important aspect of maintaining the quality of item banks is depositing new items.

\section{Depositing new items to the item bank}

In depositing new items into an item bank, we need to distinguish between large and small deposits of items into an initial item bank [3]. In the large deposit approach, item banks measuring the same construct are merged, which is similar to our earlier described a posteriori approach to item banking. For this approach to work, it is of uttermost importance to carefully compare to what extent the constructs measured by the different item banks are really the same. In the small deposits approach new, locally developed items are added to the initial item bank. These items are typically developed to suit the assessment blueprint underlying the given item bank which is in line with the a priori approach to item banking. For both large and small deposits, depositing new items into an initial item bank is similar to the procedure of linking multiple test forms, where one of these test forms is the initial item bank itself [11]. Thereby, linking generally refers to statistical methods used to establish relationships between items of different test forms [33]. A linking methodology that seems to be appropriate for purposes of small deposits of items is calibration [33]. Calibration requires only that both test forms are measuring the same construct. Unlike the equating methodology, it does not require equal reliability of test forms, which is the reason why calibration is preferred to equating when it comes to linking the scale of a shorter, less reliable form (e.g., a small set of new items) to a longer, more reliable form (e.g., initial item bank). Another slight difference between equating and item banking is that in test equating our goal is to place person measures from different forms onto the same scale, while in item banking the goal is to put the item calibrations onto the same scale [12].

Certainly, before starting a large-scale depositing study, it is recommended to conduct small-scale item try outs in order to check whether the items we intend to deposit are a valid measure of the given construct. After having collected initial evidence on the quality of the new items, Wolfe [12] proposes to implement the following Rasch-based procedure for depositing items into an item bank: (1) Select a linking design, (2) estimate item parameters and transform parameters to the same scale, (3) evaluate the quality of linking.

\section{Selecting a linking design}

Relevant literature describes many designs for linking the scales of different test forms [34]. Most of these designs eventually boil down to administering test forms that share a common set of items to different groups of examinees or to have a common group of examinees solve all the test forms that are to be linked. In practice, most often the common item approach is used [35], because typically we do not want to overburden examinees by asking them to solve multiple test forms. This particularly holds for studies in which we want to link a new test form to an initial item bank that has been calibrated at some time point in the past and access to the original sample of examinees is not possible at all.

Implementing a small deposit of items through the common-item design should proceed through following steps: (i) Choosing a set of items that will be common for the initial item bank and new test form, (ii) estimating item difficulty parameters and putting them onto the same scale, (iii) evaluating the quality of linking, and (iv) analyzing and handling linking issues. 
Linking quality largely depends on the choice of common items [36,37]. Generally, we want to choose a set of common items for which we think that it would fit the Rasch model and result in an invariant hierarchy of item difficulties across a large number of different testing situations. Also, we would want to get a similar ranking of respondents on the set of common items and on the whole item bank.

Consequently, one important consideration in choosing a set of common items is the number of common items. In the literature, the recommended optimal number of common items ranges between 15 and 25 [12], and there are some authors who suggested that in certain circumstances even as few as 2 items can be sufficient [38]. Generally, including a larger number of items contributes to more reliable linking [39]. However, this only holds if all these items have good psychometric properties, such as a good fit to the Rasch model. An important factor is also the range of difficulties of common items. Here, different recommendations exist in the literature; some recommend that the set of common items completely resembles the range of the difficulties from the item bank (minitest approach), whereas others suggest that better linking results are obtained if we use a narrower range of item difficulties (miditest approach) [37]. Somewhere, in between is the semi-miditest approach which foresees a relatively broad range of item difficulties with the extreme item difficulties excluded. When it comes to the distribution of difficulties of common items there is no conclusive recommendation from earlier research; both uniform and normal distributions showed to work well in earlier studies [40]. Taking into account that different opportunities to learn may result in violation of item difficulty invariance [30], it is reasonable to also use low DIF contrasts (for comparisons of interest) as an additional criterion in common item selection. Besides psychometric criteria, it is very important to ensure that common items represent well the assessment blueprint of the item bank (content and learning goals) $[36,37]$. Furthermore, it is important to take into account some technical factors, such as similar position of the common items in all forms that we link (e.g., test form used in the initial banking study and new test form), same picture to text positions and using secure items that had not been exposed too much through earlier studies [41].

\section{Estimating item parameters and putting them onto the same scale}

The selected set of common items together with the set of new items constitutes the new test form to be used in a depositing study, i.e., study conducted with the aim to extend the initial item bank. It is recommended that the new test form is administered to a minimum of 100 persons from the population of interest [16]. Then it is suggested to conduct a Rasch analysis of the students' answers from the depositing study and to compare the difficulties of the common items from this analysis with their difficulties from the initial item banking study [42]. One important measure in this analysis is the ratio of standard deviations for these two sets of item difficulties. If this ratio is close to 1 , the hierarchies of item difficulties from the two studies are given in units with the same substantive meaning and we can proceed with putting items onto the same scale [43]. Here, we again have two options: (i) if the depositing study is of equal priority as the initial item banking study it is recommended to proceed with the simultaneous calibration of all data from the two studies, (ii) otherwise, separate calibrations are recommended in which the difficulties of common items are fixed to the values from the test form of higher priority. Generally, fixing the difficulties of common items is safe if theory or earlier research indicates that these items should exhibit low differential item functioning (DIF) across various student samples from the given population.

After we choose and implement one of these procedures, the item difficulties from the depositing study are on the same scale as are the item difficulties from the initial item bank.

\section{Evaluating the quality of linking}

Quality of linking is primarily evaluated based on the fit of common items and invariance of their Rasch difficulties [12]. If the same items fit the Rasch model for one test form (e.g., initial item bank) but do not fit the Rasch model for another test form (i.e., new test form from the depositing study), this is a strong indicator that the remaining items from the two test forms do not measure the same construct. In that case, the equal construct requirement of calibration is not satisfied and it is not reasonable to proceed with bringing the items onto the same scale, i.e., depositing new items into the item bank. In addition, if the hierarchy of difficulties of common items changes from one to the next test form, persons with the same ability estimates have different theoretical probabilities of correctly solving some items. In other words, comparability of ability estimates from different testing occasions becomes questionable.

Generally, it is recommended to first evaluate fit and invariance for difficulties of common items [42]. Item infit and outfit indices are used to evaluate whether the common items fit the various test forms [32]. Values below 1.5 are considered as productive for measurement [44]. Invariance of difficulties of common items may be checked through a cross plot of difficulties of common items from the separate analyses of initial item banking data and depositing study data. The slope of the line-of-best fit that is close to 1 is evidence of a good item parameter stability [42]. Further, related criteria suggest that the ratio of standard deviations for the two sets of item difficulties should be between 0.88 and 1.13, and that the correlation between the two sets of difficulties should be higher than 0.94 [45]. If these conditions are not satisfied it is recommended to iteratively drop common items with large values of robust $z$, either 
until the conditions are met or until $20 \%$ of common items have been dropped [46,47]. Robust $z$ is a measure of item parameter stability for different testing occasions. It is calculated for each common item based on the following [46]:

$$
z_{R}=\frac{D-M d}{0.74 \mathrm{IQR}}
$$

where $D$ is the difference in difficulty estimates for a common item, $M d$ is the median of all difficulty differences for common items, and IQR is the interquartile range for this set of differences. Values of $z_{R}$ higher than $|1.645|$ are considered as evidence for substantial instability of the difficulty parameter [45].

Wolfe [12] provides four measures of linking quality: item-within-link fit, item-between-link fit, link-within-bank fit, and form-within-bank fit. If there are just two test forms, as is the case in our study (initial item bank and form from the depositing study), only item-within-link fit and itembetween-link fit can be calculated. Item-within-link fit is an overall or aggregated measure of common item fit and it is calculated based on the following:

$$
\mathrm{MSF}_{\mathrm{IWL}}=\frac{\sum_{i=1}^{L}\left(\mathrm{MSF}_{i j}+\mathrm{MSF}_{i k}\right)}{2 L},
$$

where $L$ is the number of common items, $\mathrm{MSF}_{i j}$ is the infit measure of common item $i$ on form $j$, and $\mathrm{MSF}_{i k}$ is the infit measure of common item $i$ on form $k$. The variance of $\mathrm{MSF}_{\mathrm{IWL}}$ is approximately equal to

$$
V=\frac{1}{L(N-1)},
$$

where $N$ is the number of respondents. The standardized $\mathrm{MSF}_{\mathrm{IWL}}$ follows the $t$ statistics and can be calculated as

$$
t_{\mathrm{IWL}}=\frac{3\left(\sqrt[3]{\mathrm{MSF}_{\mathrm{IWL}}}-1\right)}{\sqrt{V}}+\frac{\sqrt{V}}{3} .
$$

Values of $t_{\mathrm{IWL}}$ lower than 2.00 indicate that the fit aspect of linking is good.

On the other hand, linking precision and invariance of common item difficulty is quantified by the item-betweenlink fit measure which is calculated as follows:

$$
\chi_{\mathrm{IBL}}^{2}=\sum_{i=1}^{L}\left(\frac{\delta_{i k}^{\prime}-\delta_{i j}^{\prime}}{\sqrt{\mathrm{SE}_{i k}^{2}+\mathrm{SE}_{i j}^{2}}}\right)^{2},
$$

where $\delta_{i k}^{\prime}$ and $\delta_{i j}^{\prime}$ denote the difficulty of common item $i$ on the forms $k$ and $j$, respectively. Here it is assumed that these two difficulties were brought to the same scale, and SEs denote the standard errors of the two item difficulty estimates.

\section{Analyzing and handling linking issues}

In depositing studies linking quality can be jeopardized by misfitting or instability of common items. Misfitting items can be an indicator that new items do not measure the same construct as the items from the initial item bank. Before dropping these items one should carefully check whether misfit resulted from some technical issues, such as poor printing or miskeying of correct answers. We have to be particularly careful when working with small student samples where a few "strange" responses can cause one to think an item is misfitting.

More often than issues with misfit are issues related to instability of the item difficulty estimate. This instability is also known as item parameter drift. Item parameter drift (IPD) may be defined as differential functioning of items (DIF) across examinee groups associated with different testing occasions or time points $[30,48]$. In other words, persons of the same overall ability happen to have a different probability of correctly solving the same item, in different testing occasions. This change of item difficulties over time may result in issues with fairness and validity of interpretations of ability change over time [47]. However, similarly to the ordinary DIF measure, the IPD itself is not necessarily an indicator of measurement bias. Measurement bias is at hand only if the DIF is caused by a secondary construct that is not relevant for the given measurement [30]. As a consequence, it is important to reflect on possible sources of IPD [29]. Random sources of IPD are mainly related to sampling fluctuations between the compared testing occasions, as well as to linking error due to a relatively small set of common items [39]. This kind of item parameter drift may be often recognized by a nondirectional, random distribution of robust $z$ measures. Systematic sources of IPD are typically related to systematic changes of the curriculum or changes in opportunities to learn, in general. For example, changing the instruction focus from one metric system to another will change the relative difficulties of corresponding items [29]. Furthermore, lower drift is typically observed on items that focus on the application of skills than on items that focus on knowledge of specific content [49]. It has been also shown that recency of instruction in interaction with the content of common items may affect linking precision [47]. In fact, some authors claim that curriculum related achievement tests have differential validity depending on when the student takes the test and suggest test constructors to clearly specify whether their instrument is to be used for measuring immediate-after-instruction understanding or enduring understanding of concepts [30]. Finally, IPD may also result from $[7,41]$ curriculum content or skills becoming common knowledge (e.g., counting backwards and rocket launches), teaching to the test and test exposure, 
different modes or times of test administration (e.g., computer based vs paper and pencil) and different positions of items in the test forms that we are linking.

If the detected IPD is not a result of measurement bias, one can proceed in one of the following three ways [11]: (i) retaining the old item difficulty value, (ii) assigning the new difficulty value, (iii) calculation of an information weighted average difficulty. The exact choice depends on our assessment of the priority of various testing situations. If we believe that none of the different testing situations has priority, we go with calculating the information weighted average of item difficulties, based on the following:

$$
\delta_{i}=\frac{1 / \mathrm{SE}_{i 1}^{2}}{1 / \mathrm{SE}_{i 1}^{2}+1 / \mathrm{SE}_{i 2}^{2}} \delta_{i 1}+\frac{1 / \mathrm{SE}_{i 2}^{2}}{1 / \mathrm{SE}_{i 1}^{2}+1 / \mathrm{SE}_{i 2}^{2}} \delta_{i 2},
$$

where $\delta_{i}$ is the weighted average difficulty of item $i, \delta_{i 1}$ is the difficulty of item $i$ on form $1, \delta_{i 2}$ is the difficulty of item $i$ on form 2, and SEs are the corresponding standard errors.

The standard error of this information weighted average of item difficulty is calculated as

$$
\mathrm{SE}_{\delta_{i}}=\frac{1}{\sqrt{1 / \mathrm{SE}_{i 1}^{2}+1 / \mathrm{SE}_{i 2}^{2}}}
$$

Generally, it is recommended to periodically conduct item drift studies and to systematically store information about item performance in different testing occasions. Based on this tracking of item performance over time it is possible to make better decisions on the necessity of dropping an item or updating its difficulty in the item bank.

\section{RESEARCH AIM}

In this study, our aim was to demonstrate the smalldeposit approach for adding new items to an existing wave optics item bank. Particularly, we aimed to identify effective criteria for choosing linking items and to demonstrate procedures for evaluating the quality of linking and handling item parameter drift.

Combined with our initial item bank development study, this research provides a holistic picture of item banking and its potential uses in physics education research. It also provides 12 new, extensively validated wave optics conceptual questions which makes it potentially relevant for everyday teaching practice.

\section{METHODOLOGY}

\section{A. Description of the initial wave optics item bank}

When attempting to deposit new items to an item bank, first of all it is important to check whether they validly reflect the construct measured by the item bank and whether they possess satisfactory technical features (e.g., readability, clarity). Our wave optics item bank is supposed to be used for measuring physics students' understanding of wave optics. It is constructed based on a model of students' understanding of wave optics (Supplemental Material A [50]). That model describes learning goals relevant for developing competence in understanding wave optics. Within the study of developing the initial item bank of wave optics items an item pool of 65 items has been created [10]. The process of creating the item pool included many substudies such as group interviews and think alouds for checking cognitive validity, expert surveys for checking content validity, written try outs for further evaluation of cognitive validity, and achieving preliminary insight into psychometric features. However, eventually, only 35 out of these 65 items were field tested with a large student sample and 32 of the items were included into the initial wave optics item bank.

In this study, we decided to field test 12 new items from the existing item pool for inclusion into the wave optics item bank. Taking into account the fact that content validity and cognitive validity of these items were already established in the initial study by Mešic et al. [10], in this study our main task was to link the new 12 items with the 32 items that had been calibrated within the initial study.

\section{B. Description of methodological steps for depositing new items}

Our study proceeded through the following steps: (1) selecting a linking design, (2) selecting a set of common items and set of items to be deposited, (3) choosing a student sample and conducting a field test, (4) Rasch modeling and evaluating quality of linking, (5) depositing items to the bank.

\section{Selecting a linking design}

We decided to use a common item design for linking the scale from the depositing study (i.e., new test form) with the scale from the initial item banking study (i.e., initial item bank). Using a common person design was not an option, because examinees from the initial item banking study completed the survey completely anonymously.

\section{Selecting a set of common items and set of items to be deposited}

When selecting the set of common items, we took into account the following factors: (i) number of common items, (ii) fit statistics, (iii) content representativeness and DIF contrasts for groups of interest, (iv) range of item difficulties, and (v) positioning of common items.

Because of practical reasons (time limits and potential overburdening) we did not want our new test form to exceed 30 items. Consequently, we decided that our form should consist of 18 common items and 12 new items. Earlier research has suggested that this number of common items may be considered as good [12,42]. 
All items from our initial item bank had item infit and outfit statistics between 0.7 and 1.3. Thus the fit statistics was not a limiting factor at all in our process of choosing the best set of common items.

When it comes to content representativeness of the common items, we attempted to establish it by using the existing model of understanding of wave optics where all 32 calibrated items are already assigned to learning goals [10]. Then we performed a selection procedure which was similar to stratified proportional sampling. For each learning goal approximately $50 \%$ of the existing items were chosen to be common items. When choosing between items from one learning goal we compared their DIF contrasts for low versus high ability comparisons, as well as for interuniversity comparisons. Items with lower DIF contrasts were selected into the initial linking set. Later, some of these decisions related to the DIF factor were reconsidered for purposes of achieving a good item difficulty spacing and range.

In fact, earlier studies showed that it is very important that the difficulty of the common items represents well the item difficulties from the initial item bank. Our approach was somewhere between the minitest and semi-miditest approaches (Sec. II C). Since the range of item difficulties in our initial item bank was already somewhat limited $(-2.10-1.67$ logits with a mean of zero) we decided not to use a strict miditest approach. However, due to establishing optimal content representativeness, we decided not to use the easiest item from the initial item bank as a common item, because we already had a sufficient number of items related to the corresponding learning goal. We inspected the difficulties of all 32 existing items and analyzed range and spacing of difficulties for the 18 items that were chosen into the initial common item set. Then some of the previous decisions (related to the DIF factor) were reconsidered with the purpose of establishing good spacing of item difficulties. Thus we obtained our set of common items. The difficulty of the common items ranged between -1.63 and 1.67 logits (mean value $=0.02$ ). The spacing of difficulties was relatively good: $-1.63,-1.12,-0.87,-0.81,-0.58$, $-0.38,-0.32,-0.11,0.06,0.14,0.16,0.4,0.54,0.63,0.71$, $0.81,1.08,1.67$.

Next, we assembled a test form consisting of 30 items (18 common items and 12 new items) which was to be used in the depositing field study (Supplemental Material B [50]). All the questions from the student background survey were retained to be the same as in the initial item banking study. In addition, we attempted to hold constant the positions of common items in the new test form (e.g., Item $15 \mathrm{~A}$ is the 15 th item in the test, for both studies) and test form from the initial item banking study.

\section{Choosing a student sample and conducting the field test}

The initial item banking study by Mešic et al. [10] found that the items from the item bank may be used for measuring conceptual understanding of wave optics in postinstruction samples of physics students. For depositing new items into an initial item bank it is important to use a sample from the same target population [17,47]. Furthermore, as for all calibration purposes, it is recommended to have a sample size of at least 100 students [16]. Thus, our task was to find a sample of minimally 100 physics students who already learned about wave optics at university. Taking into account the small number of physics students at individual universities in Slovenia, Croatia, and Bosnia and Herzegovina, we decided to combine student samples from University of Sarajevo (Bosnia and Herzegovina), University of Rijeka (Croatia), University of Zagreb (Croatia), and University of Maribor (Slovenia). For all these universities we only included into the sample those physics students who already learned about wave optics at the university level. Eventually, 106 physics students from various years of study were included. Coverage of wave optics content largely varied between the sampled universities. While many students from the Rijeka University learned about wave optics for at least $30 \mathrm{~h}$ of lectures and $20 \mathrm{~h}$ of exercises, the students from Zagreb University covered this topic area through only $4 \mathrm{~h}$ of lectures and $3 \mathrm{~h}$ of exercises. Generally, at most of the sampled universities curricula foresee covering a wider spectrum of wave optics topics than covered in typical U.S. introductory courses of physics which follow textbooks such as Physics for Scientists and Engineers with Modern Physics by Serway and Jewett [51]. Thereby, the presentation of wave optics topics is often highly mathematized which sometimes hinders the students to focus on developing conceptual understanding. A more detailed insight into the wave physics curricula at the sampled universities can be gained by consulting the paper by Mešić et al. [10]. Thereby, it should be noted that many students from our depositing study did not finish the whole wave physics curriculum at the time of testing; i.e., most of them learned about wave optics in only one course (Table I).

Field testing was conducted at different time points for the different universities. The first testing was conducted in December 2019 with students from University of Sarajevo, and our last testing was conducted in the first week of March 2020 with students from University Zagreb. Testing has been conducted within the context of regular teaching hours; all students who on the testing day attended instruction in a selected course were included into the sample.

Table I may be used for comparing demographic features of the student samples from the depositing and initial item banking study.

It is useful to note that, in general, the two student samples are similar, but not identical. An important difference is related to the fact that in our depositing sample for even $37.8 \%$ of students more than 12 months passed between learning wave optics and taking the test. In the 
TABLE I. Characteristics of the student samples in the initial and depositing study.

\begin{tabular}{|c|c|c|c|}
\hline & $\begin{array}{c}\text { Total } \\
(N=225) \% \\
\text { of valid } \\
\text { answers }\end{array}$ & $\begin{array}{c}\text { Initial study } \\
\left(n_{1}=119\right) \% \\
\text { of valid } \\
\text { answers }\end{array}$ & $\begin{array}{c}\text { Depositing study } \\
\left(n_{2}=106\right) \% \text { of } \\
\text { valid answers }\end{array}$ \\
\hline \multicolumn{4}{|l|}{ University } \\
\hline Zagreb (UNZA) & 47.6 & 60.5 & 33.0 \\
\hline Maribor (UNMA) & 11.1 & 12.6 & 9.4 \\
\hline Sarajevo (UNSA) & 13.8 & 13.4 & 14.2 \\
\hline Rijeka (UNRI) & 25.8 & 10.1 & 43.4 \\
\hline Split (UNSP) & 1.8 & 3.4 & $\cdots$ \\
\hline \multicolumn{4}{|l|}{ Age } \\
\hline 19-20 & 23.6 & 17.3 & 30.0 \\
\hline $21-23$ & 48.9 & 48.0 & 51.3 \\
\hline $24-30$ & 27.5 & 34.7 & 18.7 \\
\hline \multicolumn{4}{|l|}{ Gender } \\
\hline Male & 52.7 & 53.4 & 51.9 \\
\hline Female & 47.3 & 46.6 & 48.1 \\
\hline \multicolumn{4}{|l|}{ Year of study } \\
\hline $1^{\text {st }}$ & 1.3 & 2.5 & $\ldots$ \\
\hline $2^{\text {nd }}$ & 33.5 & 31.1 & 36.2 \\
\hline $3^{\text {rd }}$ & 14.7 & 6.7 & 23.8 \\
\hline $4^{\text {th }}$ & 14.7 & 19.3 & 9.5 \\
\hline $5^{\text {th }}$ & 35.7 & 40.3 & 30.4 \\
\hline \multicolumn{4}{|c|}{ Time passed since learning wave optics? } \\
\hline Less than 3 months & $\quad 39.3$ & 41.5 & 36.8 \\
\hline $3-6$ months & 16.5 & 24.6 & 7.5 \\
\hline 6-12 months & 13.8 & 10.2 & 17.9 \\
\hline 12-24 months & 14.3 & 11.9 & 17.0 \\
\hline $\begin{array}{l}\text { More than } \\
24 \text { months }\end{array}$ & 16.1 & 11.9 & 20.8 \\
\hline \multicolumn{4}{|c|}{ Number of courses that covered wave optics? } \\
\hline One course & 40.4 & 31.9 & 50.0 \\
\hline Two courses & 28.4 & 32.8 & 23.6 \\
\hline $\begin{array}{l}\text { Three of more } \\
\text { courses }\end{array}$ & 31.1 & 35.3 & 26.4 \\
\hline
\end{tabular}

initial sample, there were only $23.8 \%$ of such students. In addition, in the initial study sample there were many more students who learned wave optics in multiple university courses. Finer analyses show that the main difference between the two testing occasions is related to the fact that the depositing sample, unlike the initial study sample, lacked physics teacher students from higher years at the University of Zagreb.

\section{Rasch modeling}

Judging the relative length of the logits.-In order to compare the scales from the initial item banking study and the depositing study, item difficulty estimates from separate Rasch analyses for these two studies had to be obtained. For the initial item banking study a Rasch analysis had been already conducted by Mešić et al. [10], and as a result difficulties of common items for that study were already known. Thus, we had to conduct only a Rasch analysis of the data collected in the depositing study. The recoded version of the depositing study database consisted of 106 students' answers to 30 items. We used Winsteps 4.0 software [42] for Rasch modeling. Rasch analysis of the depositing study database proceeded through the following steps:

1. We ran a Winsteps analysis which included the answers of all 106 students and all 30 items.

2. Based on Szabo's [16] recommendation, we first analyzed person fit and identified 5 persons with answering patterns that did not fit the Rasch model (i.e., person infit our outfit indices above 1.5 and negative point-biserial correlations).

3. The five persons with misfitting answering patterns were dropped from the database. Thereafter, we reran the Rasch calibration procedure which included 101 students and 30 items.

4. Now there were no more persons with negative point-biserial correlations. In addition, all the person infit indices were below 1.5 and only two persons had outfit indices slightly above 1.5 , i.e., 1.54 . We decided not to delete these two persons from the database, because of good infit indices and positive point-biserial correlations. The item infit index ranged from 0.88 to 1.16 and the item outfit index ranged from 0.86 to 1.20 . These values indicate very good item fit [44].

Taking into account that all items exhibited very good fit none of them has been dropped from our analysis. Now we had two item difficulty values for each of our 18 common items: one value from the initial item banking study and one value from this depositing study. Then we proceeded as follows:

1. We calculated the standard deviation for the set of difficulties of common items from the initial study and depositing study. Next, a ratio of these standard deviations (RSD) was calculated and it amounted to 1.15, which is above the suggested benchmark of 1.13. We also calculated the correlation coefficient (CORR) for the difficulties of common items from the two studies. It amounted to 0.83 which is below the suggested benchmark of 0.94 .

2. In line with recommendations from relevant literature, in the next step we iteratively dropped common items with the highest robust $z$ values and recalculated the RSD and CORR values.

3. For only 2 out of 18 items (16A and 4B) the robust $z$ was above the critical value of $|1.645|$ and, consequently, these items were first dropped from the linking set. However, for purposes of fulfilling the criteria on RSD and CORR measures, we decided to 


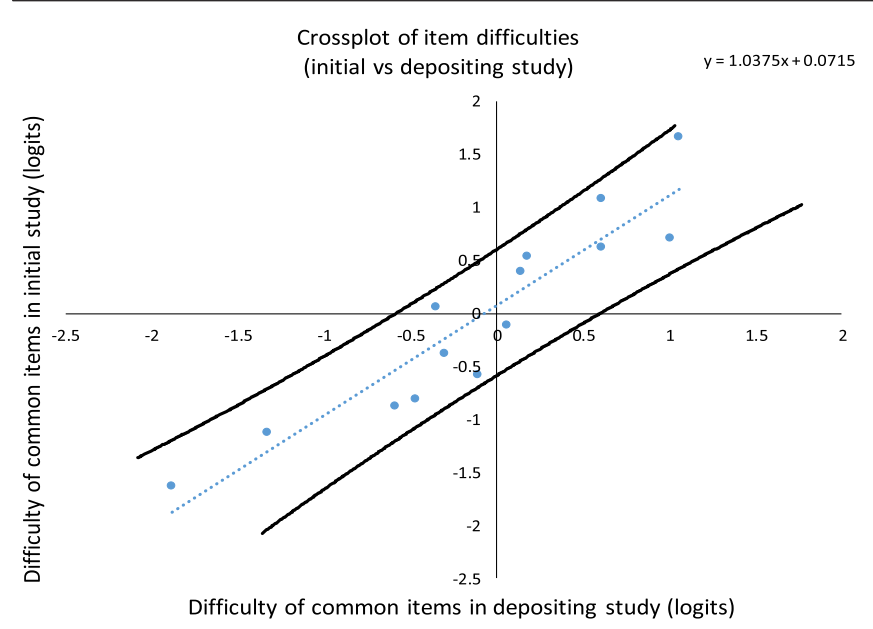

FIG. 1. Line of best fit for cross plot of difficulties of common items from the initial and depositing study, as well as a $95 \%$ confidence band are shown as evidence that difficulties of common items are invariant within the limits of measurement error.

drop another two items (20A and 11B) for which displacement values were above 0.6 [52]. After dropping 4 common items (22\% of our linking set) with the highest robust $z$ values the RSD amounted to 0.90, which is considered as acceptable and CORR amounted to 0.93 which is very close to the value of 0.94 , which is considered as acceptable (value of 1 is ideal). The robust $z$ values of the remaining 14 common items were all considerably below the critical benchmark of $|1.645|$.

4. In the cross plot of difficulties of common items from the initial and depositing study, the slope of the best fit line eventually amounted to 1.04 which is very close to 1 (Fig. 1).

According to Linacre and Wright [43], a slope of the best-fit line that is close to 1 is evidence that both tests measure in the units with the same substantive meaning; i.e., for both tests the logits are of the same length. This finding is important to proceed further with putting the item parameters onto the same scale.

Choosing an estimation procedure for putting item parameters on the same scale.-Taking into account that both of our studies (initial and depositing) were based on similar sample sizes, as well as the fact that we aim to use our instrument for all postinstruction samples, no matter the time passed between testing and instruction, we decided to take the simultaneous (concurrent) calibration approach for putting item parameters to the same scale [12]. To that end, we created a data file that included the data from both studies. It included 14 unique items from the initial item banking study, 12 unique items from the depositing study, as well as the 14 well-functioning common items. When it comes to the 4 common items which exhibited large difficulty drifts, they were also included in this database, but were considered as "statistically different" items. For example, for Item 16A there were only students' answers from the initial item banking study, and for its analog 16A2 (same item with different psychometric features) there were only students' answers from the depositing study. Consequently, our combined database consisted of 48 item variables and 217 person variables (i.e., all persons with good infit measures). This database has been used for all the Rasch analyses that followed.

Evaluating the link quality for the combined database.Our final set of common items consisted of 14 wellfunctioning items. Quality of linking primarily depends on the stability of item difficulty estimates, as well as on the extent to which items fit the Rasch model [12].

Stability of item difficulty estimates for common items was checked through analysis of CORR, RSD, and robust- $z$ measures. Difficulty measures of common items for the samples from the initial and depositing study could be obtained from a DIF analysis. Then we inspected whether some of DIF contrasts for the two testing occasions were greater than $|0.64|$ or statistically significant [42]. Finally, Wolfe's item-between-link fit index was calculated.

When it comes to the fit aspect of linking quality, we checked it by inspecting the infit and outfit indices of common items obtained from the Rasch analysis of the combined database. Furthermore, we calculated Wolfe's item-within-link fit.

Checking the unidimensionality and local independence assumptions.-For purposes of checking the unidimensionality assumption, we calculated the DETECT, ASSI, and RATIO indices [53]. Taking into account that we were interested to find whether existing items and new items belong to different dimensions, the above-mentioned indices were calculated based on the assumption of two item clusters (groupings), one being the items from the initial item bank and other being the new items. The calculation of DETECT, ASSI, and RATIO indices has been implemented with the R package "SIRT" [54]. This R analysis was based on the database of students' dichotomous responses, person ability estimates obtained from the SIRT package and information about the classification of items across clusters.

Additional evidence on unidimensionality has been collected with Bejar's [55] method. We again subdivided our combined database into new items and old items. For these clusters of items, we cross plotted their full test versus subtest item difficulties (Supplemental Material C [50]). Finally, we checked whether the slopes of best-fit lines in these cross plots are close to 1 and whether the mean squared distances to either the principal or theoretical axis are very small and approximately constant across item clusters (Fig. 2). If these criteria are fulfilled, both clusters measure the same construct [55]. 

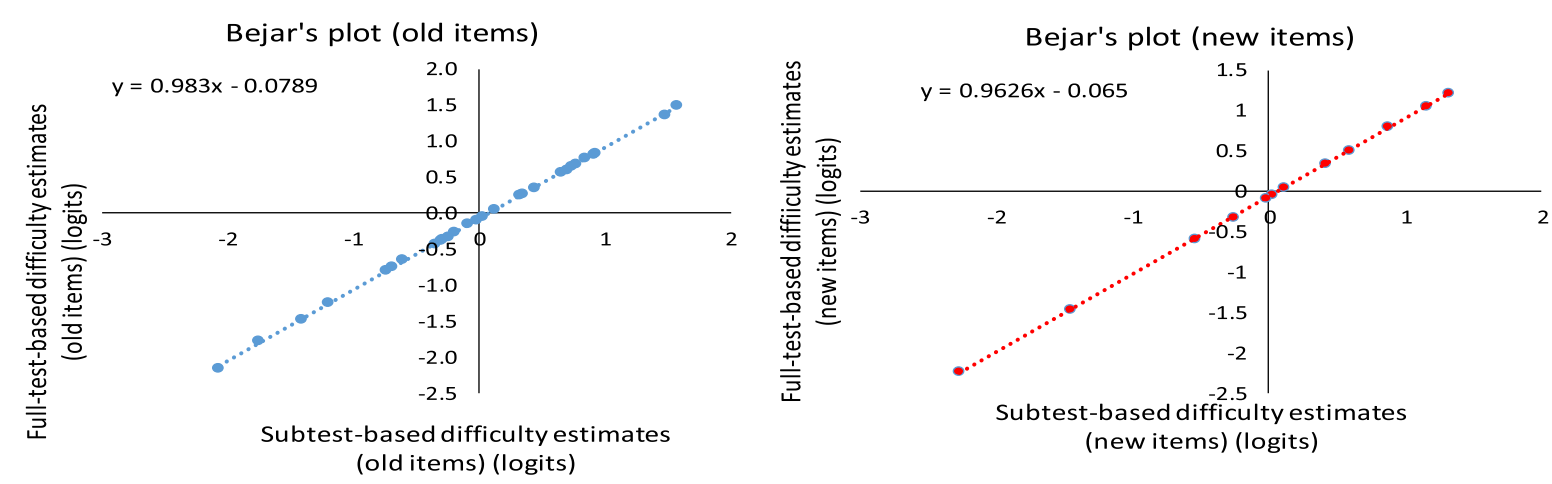

FIG. 2. Cross plots for full-test-based vs subtest-based estimates of item difficulties for "old" and "new" items.

Finally, the local independence assumption has been checked through inspection of the largest standardized correlation of Rasch residuals. If students' answering behavior on a certain item cannot be explained solely by the Rasch model there are residuals and if the residuals for a pair of items strongly correlate, then local independence is violated [42].

Analyzing item fit statistics and reliability measures.-For practical applications, it is of crucial importance that all items fit the Rasch model. To that end, we checked the item infit and outfit statistics for all our items from the combined database.

Furthermore, we were interested in the precision of measuring person and item parameter estimates. To that end we calculated the person and item reliability measures for the combined database. These measures were compared with reliability measures from separate Rasch analyses of initial banking and depositing study data.

Analyzing the Wright map (person-item map).-Before making a final decision about item depositing we wanted to check how the new items function together with old items in telling the story about students' understanding of wave optics. To that end, we created and inspected a Wright map [14]. On a Wright map persons and items are put onto the same scale measured in logits. By inspecting item difficulties we gain insight into the structure of students' understanding. If that structure is sound from a theoretical perspective than this speaks in favor of construct validity. Furthermore, the Wright map may provide us with further information about precision of measurement. When a person is perfectly aligned with an item on the map (the person and the item have the same logit measure) then for this person the probability of correct solving that item amounts to $50 \%$. For items below that item the probability of correct solution is higher than $50 \%$ and for items above that location it is lower than $50 \%$. Items that are very far from a person's location do not contribute very much to gaining information about that person's understanding of wave optics. Consequently, the relative standing of persons and items may provide us with information about the relative strengths and weaknesses of our instrument.

\section{Depositing items and handling item parameter drift}

Finally, all items that exhibited good fit were deposited into the item bank. The following information has been added for each of the items: Item ID, Rasch difficulty measure, the standard error for the difficulty measure, infit statistics, outfit statistics, point-biserial measure, item stem and response options, comments on items' history (e.g., IPD), and related goal in the model of understanding of wave optics. For the four common items that exhibited a large drift, information-weighted average difficulties were calculated.

\section{RESULTS}

\section{A. Quality of linking}

Analysis of the combined dataset revealed infit and outfit indices between 0.7 and 1.2 for all items. The standardized value of Wolfe's item-within-link fit statistic amounted to $t=-0.62$, which is lower than the critical value of $2.00[12]$.

When it comes to the stability of difficulties of common items, results of the DIF analysis for study 1 and 2 samples showed that none of the 14 common items exhibited a DIF contrast larger than $|0.64|$ and none of these contrasts proved to be statistically significant. In addition, Wolfe's item-between-link fit statistic amounted to 19.59 which was below the critical value of 22.36 .

Finally, it could be shown that RSD for the combined analysis amounted to 0.89 , CORR amounted to 0.93 and all robust $z$ values were considerably lower than the critical value of $|1.645|$.

\section{B. Unidimensionality and local independence}

For our two clusters of "old" and "new" items, results of unidimensionality checks showed that the DETECT index amounted to -0.214 , ASSI index amounted to -0.039 and RATIO index amounted to -0.102 . 
TABLE II. Squared distances between measurement points and principal or theoretical axes.

\begin{tabular}{lccc}
\hline \hline $\begin{array}{l}\text { Squared } \\
\text { distance to } \\
\text { theoretical axis } \\
\text { (old items) }\end{array}$ & $\begin{array}{c}\text { Squared } \\
\text { distance to } \\
\text { theoretical axis } \\
\text { (new items) }\end{array}$ & $\begin{array}{c}\text { Squared } \\
\text { distance to } \\
\text { principal axis } \\
\text { (old items) }\end{array}$ & $\begin{array}{c}\text { Squared } \\
\text { distance to } \\
\text { principal axis } \\
\text { (new items) }\end{array}$ \\
\hline $2.8 \times 10^{-3}$ & $3.3 \times 10^{-3}$ & $3.2 \times 10^{-5}$ & $7.7 \times 10^{-5}$ \\
\hline \hline
\end{tabular}

In line with Bejar's method, we also created cross plots of subtest versus full-test estimates of item difficulties for the old and new items (Fig. 2).

From Fig. 2 it is evident that for both the old and the new items the slope of the trend line is very close to 1 .

For both groups of items, we also calculated the average squared distance between measurement points and principal or theoretical axes (Table II).

Finally, standardized residual correlations were calculated. The two largest standardized residual correlations were found for item pairs "Item 5C-Item 4B2" $(r=0.41)$ and "Item13A-Item 16A" $(r=0.36)$.

\section{Item fit statistics and reliability}

The item infit statistics ranged from 0.86 to 1.12 and the item outfit statistics ranged from 0.71 to 1.19 . None of the standardized fit indices was outside the range from -2.0 to 2.0. Reliability estimates for the combined database analysis, as well as for the separate analyses of initial and depositing study data are provided in Table III.

\section{Wright map (person-item map)}

The Wright map for our combined analysis is shown in Fig. 3.

The range of item difficulties was from -2.24 to 1.73 , with a mean of 0 (anchored to 0 in Winsteps) and standard deviation of 0.92 . On the other hand, person abilities ranged from -1.97 to 2.46 with a mean of -0.24 and standard deviation of 0.75 . In the initial banking study item difficulties ranged from -2.10 to 1.67 , with a mean of 0 (anchored to 0 in Winsteps) and standard deviation of 0.86 . Person abilities ranged from -1.92 to 2.54 with a mean of -0.08 and standard deviation of 0.81 .

It is also useful to note that for the combined data set there was a statistically significant, weak to moderate

TABLE III. Reliability estimates for the different Rasch analyses.

\begin{tabular}{lccc}
\hline \hline & $\begin{array}{c}\text { Initial item } \\
\text { banking study } \\
\text { analysis }\end{array}$ & $\begin{array}{c}\text { Depositing } \\
\text { study } \\
\text { analysis }\end{array}$ & $\begin{array}{c}\text { Combined } \\
\text { database } \\
\text { analysis }\end{array}$ \\
\hline Item reliability & 0.93 & 0.94 & 0.95 \\
Person reliability & 0.72 & 0.56 & 0.67 \\
\hline \hline
\end{tabular}

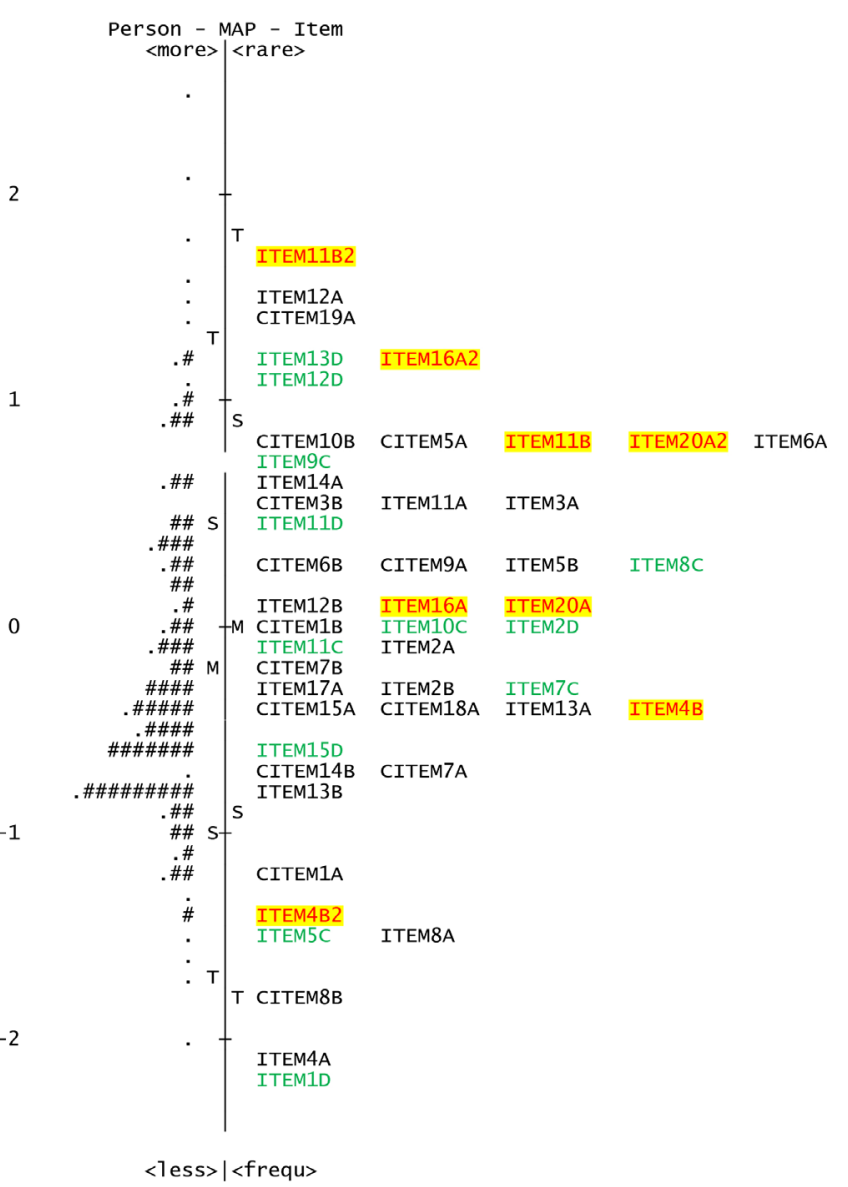

FIG. 3. Wright map for the combined analysis. Common items' labels begin with the letter "C." New items are green and four items that were dropped from the linking set are shown in yellow. For example, the label "Item 16A" describes the functioning of Item $16 \mathrm{~A}$ in the initial study and "Item 16A2" describes the functioning of the same item in the depositing study.

correlation between Rasch ability and time that passed since the student learned optics $(r=-0.24, p<0.001)$. The correlation between the number of courses in which students learned wave optics and Rasch ability proved to be nonsignificant.

\section{E. Depositing items and handling item parameter drift}

As a result of the validation procedures conducted by Mešić et al. [10], as well as linking procedures conducted in this study, all 12 new items could be deposited to the item bank.

In addition, the procedure of simultaneous calibration also resulted in updated difficulties of items from the initial item bank.

As has been pointed out earlier, four common items functioned differently for the two samples from the initial banking and depositing studies. For these items, an information weighted average has been calculated (Table IV). 
TABLE IV. Item difficulties and corresponding standard errors of four common items that functioned differently are given, together with the information weighted averages of these estimates.

\begin{tabular}{lllll}
\hline \hline & Item 16A Item 20A & Item 4B & Item 11B \\
\hline Initial item & $\delta 1=0.13$ & $\delta 1=0.09$ & $\delta 1=-0.37$ & $\delta 1=0.75$ \\
$\quad$ banking sample & SE:0.20 & SE:0.20 & SE:0.20 & SE:0.23 \\
Depositing & $\delta 2=1.21$ & $\delta 2=0.85$ & $\delta 2=-1.36$ & $\delta 2=1.73$ \\
$\quad$ sample & SE:0.26 & SE:0.24 & SE:0.23 & SE:0.32 \\
Information & $\delta=0.53$ & $\delta=0.40$ & $\delta=-0.80$ & $\delta=1.08$ \\
weighted average & SE:0.16 & SE:0.15 & SE:0.15 & SE:0.19 \\
\hline \hline
\end{tabular}

\section{DISCUSSION}

\section{A. Quality of linking}

Quality of linking largely depends on the extent to which common items fit the Rasch model, as well as on the extent to which difficulties of common items are stable, i.e., invariant [12].

We have found that the infit and outfit indices for all 14 common items from the combined database are in the range between 0.7 and 1.2 which indicates good fit [44]. Separate analyses of test forms and the obtained value for Wolfe's item-within-link fit provide additional evidence that common item fit indices are very good for both, the initial item banking study and depositing study [12].

When it comes to the stability of item difficulty estimates, in our study the values of most important statistics were mostly in line with Huynh's "golden numbers" [45]. Concretely, for the final linking set, all robust $z$ values were considerably lower than $|1.645|$, RSD was inside the range between 0.88 and 1.13 and CORR was 0.93 , which is very close to the acceptable value of 0.94 . In addition, the value of Wolfe's between-link-fit index for our linking study indicates good linking precision. It is also useful to note that analysis of the Wright map (Fig. 3) shows the 14 common items to be appropriately distributed across the whole range of item difficulties which further strengthens our confidence in linking precision across our scale.

Here we should recall that the final set of 14 common items was obtained after 4 items (22\% of all common items) were dropped from the initial linking set. Generally, it is suggested to iteratively drop instable common items either until CORR and RSD values are acceptable or until $20 \%$ of items from the initial linking set are dropped [46]. According to O'Neill, Peabody, Jin Bee Tan, and Du [52] in examinations which include less than 500 respondents typically between $10 \%$ and $30 \%$ of common items are flagged for high parameter drift. Consequently, in studies that include a relatively small number of respondents, such as is the case in our study, sometimes even $30 \%$ of items have to be unanchored.
While we can conclude that there is a broad range of evidence in favor of high quality linking for our study, it is also important to discuss possible causes of large item parameter drift for our 4 "problematic" common items. In our study three items $(16 \mathrm{~A}, 20 \mathrm{~A}, 11 \mathrm{~B})$ were shown to be differentially easier for the student sample from our initial item banking study. Concretely, the largest drifting effects were observed for students from the University of Zagreb. For the two testing occasions, the percentage of correct answers on common items on average differed by only $2.5 \%$, for the University of Zagreb samples. However, on items 16A, 20A, and 11B the differences amounted to $36 \%$, $31 \%$, and $25 \%$, respectively. Essentially, all three items were related to the misconception that diffraction fringes become narrower or smaller when we make narrower a slit or reduce the size of an aperture [56]. A possible explanation of the item parameter drift (IPD) would be that in this study the structure of the student sample was different to the first study, when it comes to the share of students from different study programmes. Consequently, the structure of learning experiences differed between the two studies. For example, unlike in the initial study, in the depositing study, the subsample from the University of Zagreb did not include physics teacher students from higher years of study who learn about conceptual aspects of physics in some teacher preparation courses. In addition, unlike in the initial study, the depositing study sample from the University of Zagreb also had no experience with laboratory exercises related to the diffraction of light on slits and apertures. Taking into account that laboratory experiments have significant potential to promote conceptual change [57], it was reasonable to expect that for the sample which had the opportunity to work through laboratory exercises the above-mentioned items were differentially easier; i.e., their relative difficulty was lower than for the student sample from the depositing study. When it comes to Item 4B, we see this result as pretty surprising. In this item, the students are asked to predict how changing the separation between three identical point sources affects the shape of the shown envelope of their wave fronts. For solving this item it is important to perform visual reasoning which is a skill and according to earlier research items focusing on skills should be more stable than those which focus on content-specific knowledge [49].

Generally, our findings seem to be in line with the fact that time passed since instruction and varying opportunities to learn may be a cause of item parameter drift [29,30,47]. Differences in opportunities to learn are often a reason why even in meticulously planned large-scale studies many items exhibit DIF contrasts for students who learned physics in line with different curricula [58].

\section{B. Unidimensionality and local independence}

With respect to unidimensionality, Robitzsch [54] suggests that DETECT values below 0.20, ASSI values below 
0.25 and RATIO values below 0.36 indicate unidimensionality. Robitzsch [54] also notes that these indices may become negative for unidimensional data. This was exactly the case for our data, providing a first piece of evidence for unidimensionality. A second piece was obtained by applying Bejar's method [55]. Subtest versus full-test crossplots, for both the new and the old items, are characterized by a slope near to one, which Bejar considers as necessary for unidimensionality of data. In other words, these results show that the inclusion of new items does not change the metric relationships between old items and vice versa. Third and last, the mean squared distances of our measurement points to the theoretical axes were very small and approximately constant across the two clusters of items. The corresponding distances to the principal axes were also very small and of the same order of magnitude. According to Bejar [55] the mean distance of items within the different clusters "to either the principal or theoretical axis" should be constant across item clusters if unidimensionality holds. Thus, we can conclude that our data are essentially unidimensional, which means that old items and new items are essentially measuring the same construct.

In terms of local independence, from the values of the largest standardized residual correlations, we could conclude that the local independence assumption holds because the observed correlations were far below 0.7 [42]. It is interesting to take a closer look at the two item pairs for which the largest standardized correlations were obtained. For the pair " $5 \mathrm{C}-4 \mathrm{~B} 2$," the result is not surprising at all because both items were related to the same physical situation in which we observe point sources of secondary waves and we have to predict how the envelope of wave fronts changes when new sources are added or separation between them is altered, respectively. Besides the dominant construct of understanding wave optics, the items require visual reasoning. Similarly, the results for the pair "13A-16A" may be explained by the fact that both of them required visual analysis of interference or diffraction patterns for the initial and modified experimental setup with the purpose of answering how the setup changed. Again, the dominant construct was understanding of wave optics, but it seems there was also a nuisance ability to visual analysis of differences [59].

\section{Item fit statistics and reliability}

All items from our combined database fit the Rasch model very well. This is an important finding because it is an additional evidence that our items measure the same ability. In fact, Wright and Panchapakesan [60] point out that in situations where guessing cannot be neglected or items are measuring different abilities, items may not fit together. In addition, many practical applications of Rasch analysis depend on the extent to which data fits the Rasch model, because model fit also affects parameter invariance [61].
As to item reliabilities, the estimates obtained for the combined data analysis, as well as for separate analyses are excellent. This means that we can have much confidence in the item difficulty hierarchy. Slightly better item reliabilities for the combined data analysis than for the separate analyses may be explained by the fact that in the combined analysis there are more persons per a common item which decreases the standard error of these item difficulty estimates. Differences in person reliabilities between the different Rasch analyses were much more pronounced. The person reliability for the sample of students from initial item banking study was considerably better than for the student sample from the depositing study for which, on average, more time passed between instruction and test taking. Taking into account that person reliabilities are interpreted in a similar manner to Cronbach's alpha coefficients, we may conclude that the person reliability for the depositing study was barely acceptable [62]. The value of person reliability for the combined analysis, was between the reliability values for the separate analyses. This could be explained by the fact that for the combined data analysis the person-item targeting was better than for the depositing study but worse than for the initial item banking study [10]. It is well known from other research that pretests often have a lower person reliability (or Cronbach's alpha) than post-tests [63]. Generally, person reliability depends on the extent to which students from the given sample have a coherent mental model of the measured construct [64]. At pretest, this is rarely the case and consequently, relatively low reliabilities are reported. Similarly, it is not a surprise that with increasing time between the end of instruction and test taking, students' mental models become less coherent and as a result consistency of measurement decreases. Consequently, the reliability of ranking persons is somewhat lower in the depositing study than in the initial item banking study. Additional explanation of results for reliability coefficients may be obtained through analysis of person-item targeting.

\section{Analysis of Wright map (person-item map)}

Based on the Wright map (Fig. 3) there is much space for improvement when it comes to person-item targeting. Most items seem to be too difficult for the average postinstruction student from universities in Slovenia, Croatia, and Bosnia and Herzegovina. It is evident that the relatively low reliability value for the depositing study is due to the fact that the average ability of the student sample for this study was below the average ability of the sample from the initial item banking study. This could be related to the fact that the depositing sample included a lower share of students from the University of Zagreb and University of Maribor who performed above average in the initial banking study. Generally, it seems that our current item bank may provide relatively reliable measures for our high-achieving, posinstruction students and less reliable measures for our 
average achievers. Concretely, it seems that the precision of measurement for postinstruction students could be significantly improved by adding more items of lower difficulties (i.e., in the range of -0.7 to -1.2 logits). When it comes to new items, it is useful to note that they have a good distribution of difficulties which means that they have the potential to improve the precision of measurement across the whole scale.

Generally, by inspection of item content, we come to the conclusion that items' potential to activate misconceptions largely influenced its difficulty. Item difficulty was lower for items that required understanding of some basic formal representations (e.g., interpreting wave fronts and sinusoids) than for items which are known to activate strong misconceptions. When it comes to the four common items that proved to function differentially for the two testing occasions, the Wright map nicely shows that 3 out of 4 problematic items were differentially easier for the student sample from the initial study. This could be mainly related to earlier discussed opportunity to learn differences for the Zagreb subsamples from the two testing occasions.

\section{E. Depositing items and handling item parameter drift}

The fact that all 12 new items had a very good fit statistics and that they, in general, proved to nicely work together with other items in describing our construct, resulted in the decision to deposit all these items to our wave optics item bank. In a posteriori studies that aim for joining items from different assessment instruments into a single item bank the number of items that have to be dropped due to misfit is often much larger [20]. This could be due to the fact that these different assessment instruments are after all based on measurement goals that are more or less different across the instruments. This increases the probability of items tapping different abilities, which leads to misfit [60]. Consequently, in our opinion, in all approaches to item banking, it is important to fix a single "model of the construct." In other words, it is important to fix a set of learning goals to which items are to be assigned, either by the development of new items specially tailored to suit these goals or by recognizing potential candidates in existing instruments that measure a very similar construct.

The four items that exhibited large parameter drift were deposited with their information weighted average difficulty and standard error. We did not decide to drop these items completely, because they exhibited good fit in both testing occasions. The reason for averaging their difficulties from the initial and depositing study is that we want to use our items to measure conceptual understanding of all postinstruction physics students, independent of time passed between instruction and test taking.

\section{F. Limitations of the study}

Our item bank is limited to being used for measuring conceptual understanding of wave optics in post-instruction samples of physics students. For universities that had not been included in our banking studies, additional evidence has to be collected on whether the established hierarchy of item difficulties also holds for their students. In our opinion, this is not a specific limitation of our item bank, but all (physics education) item banks or instruments have to be cross-validated (or recalibrated) prior to deciding whether it is appropriate to use them for measuring a particular construct in a particular target population [18]. Here a population is primarily defined by learning experiences of its members and it should be noted that even preinstruction physics students may not belong to the same population as post-instruction physics students $[65,66]$.

In our study, the calibration of item difficulties has been performed on the combined database. An advantage of this approach is that both of our studies are given equal importance and that common items are now estimated with smaller standard error. A possible disadvantage of this concurrent calibration approach is that all item difficulties are updated. Generally, this may be an issue if our aim is to use the item bank for assessing growth with time, because changing difficulty measures complicates comparing ability measures obtained at different time points. However, the wave optics item bank from our study is still in the stage of development and at this moment it is not used for growth measurement.

Finally, it should be noted that our item difficulty estimates are based on a relatively small number of students. Although the number of 100 students is considered sufficient for Rasch analyses, certainly using larger, representative samples would reduce the standard error of difficulty estimates and improve their stability. Furthermore, in our study, only a small number of items (12) has been deposited. For depositing a larger number of items in a single study, more test forms and more sets of linking items would have to be used. This was not possible to implement in our study due to the limited number of items in the initial item bank and due to limited access to a larger number of postinstruction physics students.

\section{CONCLUSION}

Item banks may help us to substantially improve the quality of educational measurement. For securing that improvement it is important to continually reflect on the extent to which our item bank mirrors the measured construct. A better and more reliable mirroring of the measured construct can be achieved through periodic removing of obsolete items and deposits of valid items.

In this study, we described in detail the procedure of depositing new items into an initial item bank. It has been shown that linking new and old items through a common item design may work well if following criteria for choosing common items are taken into account:

- Anchor length: It is recommended that the initial common item set contains between 15 and 25 items. 
- Fit indices: All common items have to be characterized by good fit.

- Range of difficulties: The difficulties of common items should represent well the range of item difficulties in the item bank, with a good spacing of these difficulties along the logit scale. Extremely easy or difficult items should not be included if the standard error of their measurement is very high.

- Content representativeness: Common items should represent well the test blueprint, i.e., for content areas or learning goals that are given more weight, more common items should be selected.

- Position in the test form: Position of common items should be very similar in the initial item banking study and depositing study.

- DIF contrasts: Items with lower DIF contrasts for comparisons of interest (e.g., interuniversity comparisons, high ability vs low ability) should be preferred. In addition to identifying a set of effective criteria for choosing linking items, our study shows that it is recommended to conduct the depositing studies with a student sample that shares similar learning experiences with the sample from the initial item banking study. All samples should be from the same target population and similar time points for testing should be chosen (e.g., immediate after instruction). Ideally, a large, random sample of students should be selected. Otherwise, the learning experiences for samples from different testing occasions may substantially differ (e.g., largely different emphasis on certain parts of wave optics), which can lead to difficulties of common items being not invariant and linking precision being lowered.

In our study, the set of common items proved to be characterized by fair linking precision and very good fit in both test testing occasions. Multidimensionality and local independence studies indicate that Rasch model assumptions were met and reliability measures were fair. After collecting corresponding evidence on validity, reliability, and linking quality, we could decide to extend our wave optics item bank by an additional 12 items. The extended item bank may be used for creating comparable test forms that measure understanding of wave optics, as well as for sparking discussions about conceptual aspects of this area of physics.

In our future research we will attempt to further extend our item bank and extensively study person-item targeting for various subsamples of students and curricular contexts. More items of below average difficulty will be added for purposes of increasing person reliability.

\section{ACKNOWLEDGMENTS}

This work has been supported in part by the University of Rijeka under Project No. uniri-pr-prirod-19-5, as well as by the Ministry of Science and Education of Republic of Croatia under the bilateral CRO-SAD Project No. 2/2019.
[1] B. D. Wright and S. R. Bell, Item banks: What, why, how, J. Educ. Measure. 21, 331 (1984).

[2] Licensure Testing: Purposes, Procedures, and Practices, edited by J. C. Impara (Buros, Lincoln, 1995).

[3] L. M. Rudner, Item banking, Pract. Assess. Res. Eval. 6, 4 (1998).

[4] D. J. Weiss, Item banking, test development, and test delivery, in APA Handbooks in Psychology. APA Handbook of Testing and Assessment in Psychology, Vol. 1. Test theory and testing and assessment in industrial and organizational psychology, edited by K.F. Geisinger, B. A. Bracken, J. F. Carlson, J.-I. C. Hansen, N. R. Kuncel, S. P. Reise, and M. C. Rodriguez (American Psychological Association, Washington, DC, 2013), pp. 185-200.

[5] D. Cella, R. Gershon, J.-S. Lai, and S. Choi, The future of outcomes measurement: Item banking, tailored shortforms, and computerized adaptive assessment, Qual. Life Res. 16, 133 (2007).

[6] M. J. Kolen and R. L. Brennan, Test Equating, Scaling, and Linking (Springer, New York, 2004).

[7] M. J. Linacre, Computer-adaptive testing: A methodology whose time has come. Mesa memorandum No. 69, in
Development of Computerised Middle School Achievement Test, edited by S. Chae, U. Kang, E. Jeon, and J. M. Linacre (Komesa Press, Seoul, 2000).

[8] J. B. Bjorner, C.-H. Chang, D. Thissen, and B. B. Reeve, Developing tailored instruments: Item banking and computerized adaptive assessment, Qual. Life Res. 16, 95 (2007).

[9] M. Planinić, W. J. Boone, A. Sušac, and L. Ivanjek, Rasch analysis in physics education research: Why measurement matters, Phys. Rev. Phys. Educ. Res. 15, 020111 (2019).

[10] V. Mešić, K. Neumann, I. Aviani, E. Hasović, W. J. Boone, N. Erceg, V. Grubelnik, A. Sušac, Dž. Salibašić Glamočić, M. Karuza, A. Vidak, A. Alihodžić, and R. Repnik, Measuring students' conceptual understanding of wave optics: A Rasch modeling approach, Phys. Rev. Phys. Educ. Res. 15, 010115 (2019).

[11] R. J. Mead, Basic ideas in item banking, in Annual Meeting of the National Council on Measurement in Education, Los Angeles, CA (National Council on Measurement in Education, 1981).

[12] E. W. Wolfe, Equating and item banking with the Rasch model, J. Appl. Meas. 1, 409 (2000). 
[13] X. Liu, Using and Developing Measurement Instruments in Science Education: A Rasch Modeling Approach (Information Age Publishing, Inc., Charlotte, NC, 2010).

[14] W. J. Boone, J. R. Staver, and M. S. Yale, Rasch Analysis in the Human Sciences (Springer Science and Business Media, New York, 2013).

[15] C. D. Vale, Computerized item banking, in Handbook of Test Development, edited by S. M. Downing and T. M. Haladyna (Lawrence Erlbaum Associates, Mahwah, NJ, 2006), pp. 261-285.

[16] G. Szabo, Applying Item Response Theory in Language Test Item Bank Building (Peter Lang, New York, Berlin, 2008).

[17] J. Rost, Lehrbuch Testtheorie, Testkonstruktion (Huber, Bern, 1996).

[18] B. Marcus and M. Buhner, Grundlagen der Testkonstruktion (FernUniversitat, Hagen, 2009).

[19] K. Pesudovs, Item banking: A generational change in patient-reported outcome measurement, Optom.Vis. Sci. 87, 285 (2010), https://journals.lww.com/optvissci/ Abstract/2010/04000/ Item_Banking_A_Generational_Change_in.10.aspx.

[20] A. B. Smith, R. Rush, G. Velikova, L. Wall, E. P. Wright, D. Stark, P. Selby, and M. Sharpe, The initial development of an item bank to assess and screen for psychological distress in cancer patients, Psychooncology 16, 724 (2007).

[21] J. N. Njiru and J. Romanoski, Development and calibration of physics items to create an item bank, using the Rasch measurement model, Int. J. Learn.: Annu. Rev. 14, 19 (2007).

[22] P. M. Sadler, H. Coyle, N. C. Smith, J. Miller, J. Mintzes, K. Tanner, and J. Murray, Assessing the life science knowledge of students and teachers represented by the K8 national science standards, CBE Life Sci. Educ. 12, 553 (2013).

[23] M. Cupani, T. C. Zamparella, G. Piumatti, and G. Vinculado, Development of an item bank for the assessment of knowledge on biology in Argentine university students, J. Appl. Meas. 18, 360 (2017).

[24] J. Mestre, J. Morphew, and G. Gladding, Learning from different styles of animated solutions among low performing participants, in Proceedings of the 2015 Physics Education Research Conference, College Park, MD (AIP, New York, 2015), pp. 223-226.

[25] E. Ene and B. J. Ackerson, Assessing learning in small sized physics courses, Phys. Rev. Phys. Educ. Res. 14, 010102 (2018).

[26] J. Han, L. Bao, L. Chen, T. Cai, Y. Pi, S. Zhou, Y. Tu, and K. Koenig, Dividing the Force Concept Inventory into two equivalent half-length tests, Phys. Rev. ST Phys. Educ. Res. 11, 010112 (2015).

[27] Y. Xiao, J. C. Fritchman, J. Y. Bao, Y. Nie, J. Han, J. Xiong, H. Xiao, and L. Bao, Linking and comparing short and fulllength concept inventories of electricity and magnetism using item response theory, Phys. Rev. Phys. Educ. Res. 15, 020149 (2019).

[28] R. M. Anzaldua, Item banks: What, where, why and how, https://eric.ed.gov/?id=ED462435 (2002).

[29] B. A. Dunya, Item parameter drift in computer adaptive testing due to lack of content knowledge within sub-populations, Ph.D. thesis, University of Massachusetts, 2017.

[30] T. M. Sukin, Item parameter drift as an indication of differential opportunity to learn: An exploration of item flagging methods and accurate classification of examinees, Ph.D. Thesis, University of Massachusetts, 2010.

[31] T. W. G. van der Meer and E. Ouattara, Putting political back in political trust: An IRT test of the unidimensionality and cross-national equivalence of political trust measures, Qual. Quant. 53, 2983 (2019).

[32] T. Bond and C.M. Fox, Applying the Rasch Model: Fundamental Measurement in the Human Sciences (Routledge, London, 2015).

[33] K. Ryan and L. Shepard, The Future of Test-Based Educational Accountability (Routledge, London, 2010).

[34] R. K. Hambleton, H. Swaminathan, and H. J. Rogers, Fundamentals of Item Response Theory (Sage, Thousand Oaks, CA, 1991).

[35] E. Muraki, C. M. Hombo, and Y. W. Lee, Equating and linking of performance assessments, Appl. Psychol. Meas. 24, 325 (2000).

[36] L. W. Klein and D. Jarjoura, The importance of content representation for common item equating with nonrandom groups, J. Educ. Measure. 22, 197 (1985).

[37] S. Sinharay and P. W. Holland, Is it necessary to make anchor tests miniversions of the tests being equated or can some restrictions be relaxed?, J. Educ. Measure. 44, 249 (2007).

[38] M. S. Wingersky and F. M. Lord, An investigation of methods for reducing sampling error in certain IRT procedures, Appl. Psychol. Meas. 8, 347 (1984).

[39] D. Budescu, Efficiency of linear equating as a function of the length of the anchor test, J. Educ. Measure. 22, 13 (1985).

[40] S. Born, A. Fink, C. Spoden, and A. Frey, Evaluating different equating setups in the continuous item pool calibration for computerized adaptive testing, Front. Psychol. 10 (2019).

[41] J. Ryan and F. Brockmann, A Practitioner's Introduction to Equating with Primers on Classical Test Theory and Item Response Theory (Council of Chief State School Officers, Washington, DC, 2009).

[42] J. M. Linacre, A Users guide to Winsteps: Rasch-model computer programs (MESA Press, Chicago, IL, 2017).

[43] J. M. Linacre and B. D. Wright, The "length" of a logit, Rasch Meas. Trans. 3, 54 (1989), https://www.rasch.org/ $\mathrm{rmt} / \mathrm{rmt} 32 \mathrm{~b} . \mathrm{htm}$.

[44] B. D. Wright and J. M. Linacre, Reasonable mean-square fit values, Rasch Meas. Trans. 8, 370 (1994), https://www .rasch.org/rmt/rmt83b.htm.

[45] A. M. W. Rawls, The Importance of Test Validity: An Examination of Measurement Invariance across Subgroups on a Reading Test (University of South Carolina, Columbia, SC, 2009).

[46] H. Huynh and P. Meyer, Use of robust $z$ in detecting unstable items in item response theory models, Pract. Assess. Res. Eval. 15, 1 (2010), https://scholarworks.umass.edu/cgi/ viewcontent.cgi? article $=1228 \&$ context $=$ pare.

[47] A. Hardy, Investigating how equating guidelines for screening and selecting common items apply when 
creating vertically scaled elementary mathematics, Ph.D. thesis, Brigham Young University, Provo, UT, 2011.

[48] H. Goldstein, Measuring changes in educational attainment over time: Problems and possibilities, J. Educ. Measure. 20, 369 (1983).

[49] K. Y. Chan, F. Drasgow, and L. L. Sawin, What is the shelf life of a test? The effect of time on the psychometrics of a cognitive ability test battery, J. Appl. Psychol. 84, 610 (1999).

[50] See Supplemental Material at http://link.aps.org/ supplemental/10.1103/PhysRevPhysEducRes.17.010105 for a more detailed description of the model of understanding wave optics (A), the survey that has been administered in the depositing study (B), and for more details about Bejar's method of checking for unidimensionality (C).

[51] R. A. Serway and J. W. Jewett, Physics for Scientists and Engineers with Modern Physics (Cengage Learning, Independence, KY, 2013).

[52] T. O'Neill, M. Peabody, R. J. B. Tan, and Y. Du, How much item drift is too much?, Rasch Meas. Trans. 27, 1423 (2013), https://www.rasch.org/rmt/rmt273a.htm.

[53] J. Zhang, A procedure for dimensionality analyses of response data from various test designs, Psychometrika 78, 37 (2013).

[54] A. Robitzsch, sirt: Supplementary item response theory models. $\mathrm{r}$ package version 3.9-4, https://CRAN.R-project .org/package=sirt (2020).

[55] I. I. Bejar, A procedure for investigating the unidimensionality of achievement tests based on item parameter estimates, J. Educ. Measure. 17 (1980).

[56] V. Mešić, A. Vidak, E. Hasović, and M. Dekić, University students' ideas about the role of the aperture and laser beam dimensions in formation of diffraction patterns, Eur. J. Phys. 40, 055701 (2019).
[57] M. Baser, Fostering conceptual change by cognitive conflict based instruction on students' understanding of heat and temperature concepts, Eurasia J. Math. Sci. Technol. Educ. 2, 96 (2006).

[58] V. Mešić, Identifying country-specific cultures of physics education: A differential item functioning approach, Int. J. Sci. Educ. 34, 2483 (2012).

[59] P. W. Holland and H. Wainer, Differential Item Functioning (Routledge, London, 1993).

[60] B. Wright and N. Panchapakesan, A procedure for sample-free item analysis, Educ. Psychol. Meas. 29, 23 (1969).

[61] T. H. Nguyen, H. R. Han, M. T. Kim, and K. S. Chan, An introduction to item response theory for patient-reported outcome measurement, The Patient - Patient-Centered Outcomes Research 7, 23 (2014).

[62] A. Bowling, Techniques of questionnaire design, in Handbook of Health Research Methods: Investigation, Measurement and Analysis, edited by A. Bowling and S. Ebrahim (Open University Press, Maidenhead, 2005), pp. 394-428.

[63] D. Bacon, The contributions of reliability and pretests to effective assessment, Pract. Assess. Res. Eval. 9, 1 (2004), https://scholarworks.umass.edu/cgi/viewcontent .cgi article $=1136 \&$ context $=$ pare .

[64] K. S. Taber, The use of Cronbach's alpha when developing and reporting research instruments in science education, Res. Sci. Educ. 48, 1273 (2018).

[65] L. L. Cook, D. R. Eignor, and H. L. Taft, A Comparative Study of Curriculum Effects on the Stability of IRT and Conventional Item Parameter Estimates (RR-85-38) (Educational Testing Service, Princeton, NJ, 1985).

[66] L. L. Cook, D. R. Eignor, and H. L. Taft, A comparative study of the effects of recency of instruction on the stability of IRT and conventional item parameter estimates, J. Educ. Measure. 25, 31 (1988). 